\title{
Determinants of Feedback Retention in Soccer Players
}

\author{
by

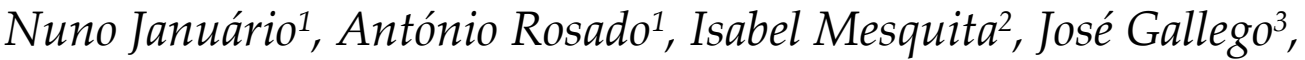 \\ José M. Aguilar-Parra
}

This study analyzed soccer players' retention of coaches' feedback during training sessions. We intended to determine if the retention of information was influenced by the athletes' personal characteristic (age, gender and the sports level), the quantity of information included in coach's feedback (the number of ideas and redundancy), athletes' perception of the relevance of the feedback information and athletes' motivation as well as the attention level. The study that was conducted over the course of 18 sessions of soccer practice, involved 12 coaches ( 8 males, 4 females) and 342 athletes (246 males, 96 females), aged between 10 and 18 years old. All coach and athlete interventions were transposed to a written protocol and submitted to content analysis. Descriptive statistics and multiple linear regression were calculated. The results showed that a substantial part of the information was not retained by the athletes; in $65.5 \%$ of cases, athletes experienced difficulty in completely reproducing the ideas of the coaches and, on average, the value of feedback retention was $57.0 \%$. Six variables with a statistically significant value were found: gender, the athletes' sports level, redundancy, the number of transmitted ideas, athletes' perception of the relevance of the feedback information and the athletes' motivation level.

Key words: Soccer players, coach feedback, information retention, motivation and attention level.

\section{Introduction}

The effectiveness of the coach's instructional process depends on how the athletes process the information provided by the coach. The active role of the learners has been highlighted in the paradigm of the mediating processes (Doyle, 1986). The active processing of instructions is a determinant factor in the learning process (Anderson, 1969), meaning that information can be actively created by the receiver (Badzinski and Gill, 1994) and the learners' level of cognitive effort influences the quality of practice of the lesson (Magill, 1993). The athletes' retention of the information transmitted by coaches by means of feedback (FB) is essential to improve performance.

Providing feedback is one of the most important instructional strategies; supplying athletes with informative feedback about their performance is crucial in the instructional process (Clemente et al., 2013; Gagné, 1985; Siekanska et al., 2013). Feedback offers the learners informative critiques of their performance to show them how to improve current skill defects and correct misconceptions or errors (Hattie and Timperley, 2007).

Receiver selectivity theory (McCroskey and Richmond, 1996) proposes that the facility to recall information depends on the extent to which receivers engage with and understand the communicator's messages, giving the athletes the ability to choose whether or not to respond to that

\footnotetext{
1 - University of Lisbon, Faculty of Human Kinetics, Portugal.

2 - Faculty of Sport, University of Porto, Portugal.

3 - Faculty of Education, University of Almería, Spain.

4 - Faculty of Psychology, University of Almería, Spain.
} 
information. McCroskey and Richmond (1996) considered that selective perception was the process of attributing meaning to messages, which suggests athletes must dynamically interpret messages to create meaning and stimulate comprehension (Badzinski and Gill, 1994).

To make FB useful, it should not only be received, comprehended and reproduced, but also accepted (Januário et al., 2006; Mesquita et al., 2008). Research results show that mediating factors, such as attention, retention and memory capacity, influence athlete's performance (Bandura, 1986).

Furthermore, perceived usefulness and ease of use are basic determinants of information retention (Davis, 1989). Oleto (2006) also refers to the importance of relevance and acceptance of the information as a qualifier of the FB instructional process. Another variable that has been shown to influence retention of feedback is the athletes' motivation level (Nideffer, 1995) as there is a relationship between the latter and mental effort and concentration.

Studies about the retention process of instructional information in the field of sport activities have mainly considered the influence of variables related to the characteristics of the information transmitted (extension, the number of ideas, the objective and the form) and athletes' characteristics (age, gender, a school level and a practice level).

Research on this particular area in physical education (Carreiro da Costa et al., 1998; Cloes et al., 1990, 1991; Januário et al., 2006) and coaching (Mesquita et al., 2008; Rosado et al., 2008) showed that considerable part of the information was not retained by the students or athletes and demonstrated that larger quantities of information transmitted by teachers or coaches made retention more difficult for athletes and students (Mesquita et al., 2008; Rosado et al., 2008; Swalus et al., 1991).

On the other hand, the effect of athletes' demographic characteristics on the instructional retention process is not completely clear. The retention of information did not differ according to the athletes' age (Mesquita et al., 2008; Rosado et al., 2008), students' age (Januário et al., 2006) or the school level (Januário et al., 2006; Rosado et al., 2008). However, in the training setting, the athletes' experience level was referenced as a differentiated variable (Rosado et al., 2008) having more experienced players retaining more ideas.

As for the effects of the variables related to the information transmitted, namely its objective and the way in which it is given, they have been shown to influence the students' retention level (Carreiro da Costa et al., 1996, 1998; Januário et al., 2006), however, the results found in various studies are contradictory. The literature shows that substantial part of the information is not retained but, more importantly, the influence of variables such as the context, athletes' characteristics and an instructional approach method (namely structure and nature) is not completely known due to a lack of data or contradictory results.

Indeed, only few studies have assessed the determinants of athletes' retention of feedback and there is a need to expand the research in this area. So far, the variables related to the retention of information have been studied separately, yet a more dynamic approach is needed in order to discover which contribute most to retention (or whether they do so) based on the use of dynamic models (such as regression analysis). This new approach could represent a major breakthrough in efforts to address this issue. Studies about the information retention process can help coaches to have a better understanding about the information retained by athletes, thereby optimizing instructional procedures and allowing reflection upon the instructional strategies.

In this study we focused on the athletes' retention of coaches' feedback during soccer practice sessions, analyzing the gap between the information that the coach transmitted and that which the athlete retained. An in-depth analysis was conducted on whether retention varied in relation to variables such as athletes' demographic characteristics, the structure and nature of the information, athletes' acceptance of the feedback and the athletes' motivation and attention level.

\section{Material and Methods}

\section{Participants}

The study that was conducted over the course of 18 soccer practice sessions, involved 12 coaches ( 8 males, 4 females, all with more than 5 years of professional experience) and 342 athletes 
(246 males, 96 females), aged between 10 and 18 years old. The athletes' sports level was as follows: 48 athletes were classified as having a very low level, 118 low, 112 high and 64 very high. This classification was based on the participants' level of competition, namely athletes playing at a local level, regional level, national level and international level. The observed instructional episodes involved 1728 FB information units emitted by the coaches during the training sessions.

\section{Measures}

The interview was designed to capture the specified domain according to the theoretical framework. Content validity was assessed through a qualitative approach based on the expert review, involving three sport researchers from two different universities. Each expert received an e-mail containing the purpose of this study, an explanation of the procedures, a detailed description of the constructs and the list of questions proposed. There was complete agreement between the experts in this validation procedure and simply minor adjustments were made. Also, a pre-test with the refined interview was conducted on a sample from the target population, based on an application in a pilot study in which five lessons were used to see if the questions were easily understood and presented in an acceptable manner. In addition, there was preliminary training on observation and codification involving two members of the research team and during this training phase there was a discussion of the statements relating to each category of the feedback analysis system. The reliability of the observations was assured by an inter-observer and intra-observers' agreement, with a three week interval, from Bellack's percentage of the agreement formula (1966, as cited by Van der Mars, 1989). Twenty percent of total instruction events were analyzed, considering that a minimum value of $10 \%$ was necessary to evaluate reliability (Tabachnick and Fidell, 1989). The lower values that we found were $93 \%$ for the inter-observer agreement and $95 \%$ for the intra-observers, which represented a strong agreement. Fleiss (1981) indicated scores greater than $75 \%$ to show adequate reliability.

\section{Procedures}

After explaining the aims of the study and receiving the athletes or parents and coaches' informed consent, the training sessions were videotaped and instructions given during the sessions were recorded on an audio and video recorder. Data was collected in a normal training environment, under normal conditions of the training process. During the session, and after coaches' feedback, the athlete was approached by a researcher and a brief recall interview was given. The interview was designed to capture athletes' recall in relation to the coaches' feedback, asking the following questions: What did your coach say? Do you mind repeating this in your own words? After that, four questions about the athletes' perception of the relevance and acceptance of feedback, the task motivation level and the athletes' attention level were evaluated using a five point scale (1- very low; 5- very high).

In this study we examined different types of feedback transmitted by the coach without considering a specific type of feedback (i.e., motivational versus instructional based performance). All coaches' and athletes' interventions were then transposed to a written protocol, which was then submitted to content analysis. No athlete was inquired during a time of emotional instability and no question was considered particularly intrusive. The protocol complied with the requirements of the Declaration of Helsinki and received the approval of the Ethics Committee of the Faculty of Human Kinetics of the University of Lisbon.

\section{Statistical Analysis}

A descriptive analysis was performed to obtain frequencies, means, standard deviations, minimal and maximal values. Multiple linear regression was also conducted, using a hierarchical model, considering as a dependent variable the retention of feedback (measured by the ratio between the ideas transmitted by the coach and those reproduced by athletes) and three independent variable groups: variables related to the athletes' characteristics (age, gender and a sports level); variables related to feedback (a number and redundancy or repeated ideas); variables related to athletes' perception of the attention level, acceptance of the coaches' feedback and the athletes' task motivation level. The requirements to use multiple linear regression were verified. Data was analyzed using SPSS V.19 software. The level of significance was set at $p$ $\leq 0.05$. 


\section{Results}

A descriptive analysis of the feedback transmitted by coaches (Table 1) shows that the total number of ideas transmitted (total number of ideas $=$ number of different ideas transmitted + number of repeated ideas) by coaches per feedback ranges between 1 and 39, with an average of 5.05 ideas (information units). The average of different ideas transmitted (not considering the repeated ideas) was 3.96 (range between 1 and 23 different ideas). The length of the information transmitted by coaches ranges between 1 and 250 words, with the average being 28.91 words per feedback transmitted.

The results showed that substantial part of the information was not retained; in $65.50 \%$ of the feedback there were difficulties to completely reproduce the coaches' ideas and only $34.50 \%$ of the feedback was completely retained. On the average, the value of the feedback retention was $57.02 \%$.

Globally, acceptance of the FB by athletes was very high $(\mathrm{M}=4.63 ; \mathrm{SD}=0.56)$ and the athletes' attention level with regard to the FB was also very high $(\mathrm{M}=4.23$; $\mathrm{SD}=0.60)$; the same for the athletes' task motivation level $(\mathrm{M}=4.62 ; \mathrm{SD}=$ 0.54).

Multiple linear regression (Table 2) shows that the model was significant $(\mathrm{F}(8.333)=18.962$; $\mathrm{R}^{2} \mathrm{a}=0.296 ; p<0.1$ ) and explained $29.60 \%$ of the feedback retention. Six variables with statistical significance were found: gender, the athletes' sports level, redundancy, the number of transmitted ideas, athletes' perception of the relevance of the feedback and the athletes' motivation level.

Considering variables related to the athletes' characteristics, gender and the athletes' sports level significantly influenced the retention of feedback. Athletes with a higher sports level showed high values of feedback retention and the male group presented higher values of feedback retention than female athletes. The athletes' age was not a discriminate variable of feedback retention.

The number of different ideas transmitted by the coach and the redundancy (the number of repeated ideas) of the feedback significantly influenced the retention of feedback. When the number of transmitted ideas decreased, the coherence of information increased and athletes presented better retention than when the feedback redundancy was higher.

The athletes' task motivation level also affected retention of the feedback information: athletes with a higher task motivation level presented a lower level of retention. Also, the athletes' acceptance of the feedback information affected retention of feedback (athletes with a higher level of acceptance presented higher levels of retention). On the other hand, the athletes' attention level was not a discriminate variable of feedback retention.

\begin{tabular}{|c|c|c|c|c|}
\hline \multicolumn{5}{|c|}{$\begin{array}{c}\text { Table } 1 \\
\text { Descriptive analysis of the coherency of information retained by athletes, } \\
\text { length of information, the total number of ideas and the different ideas transmitted. }\end{array}$} \\
\hline Variable & Average & $S D$ & Max. & Min. \\
\hline coherency of information & 57.02 & 25.33 & 100 & 0 \\
\hline length of information & 28.91 & 22.94 & 1 & 250 \\
\hline total number of ideas & 5.05 & 3.61 & 39 & 1 \\
\hline different ideas transmitted & 3.96 & 2.51 & 23 & 1 \\
\hline \multicolumn{5}{|c|}{$\begin{array}{l}\text { Total number of ideas }=\text { number of different ideas transmitted } \\
r \text { of repeated ideas. Different ideas transmitted }=\text { not considering the repeated ideas. }\end{array}$} \\
\hline
\end{tabular}




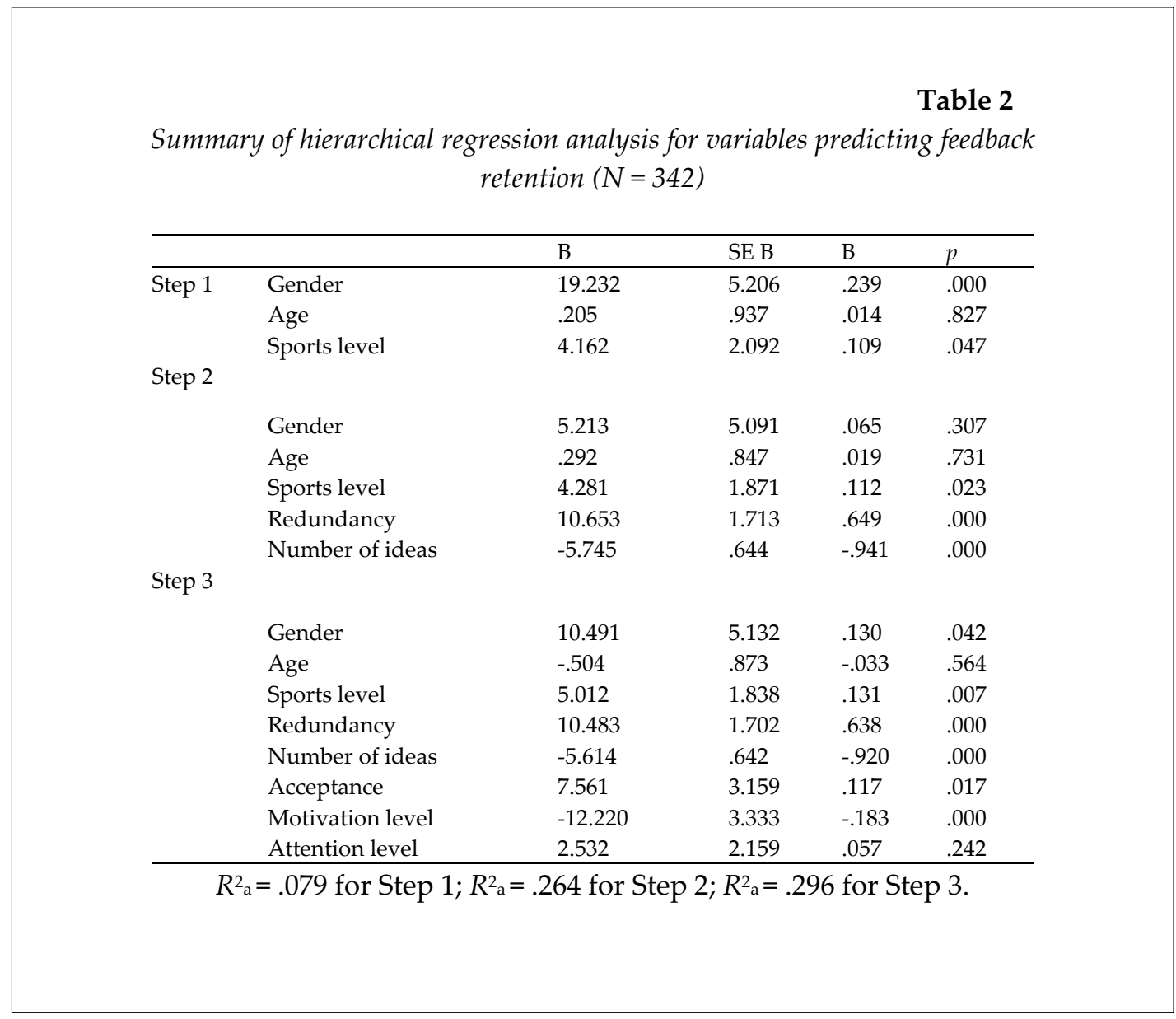

\section{Discussion}

The results of the present study indicate that substantial part of the feedback information was not retained by the athletes. Only one third of athletes completely reproduced the ideas included in feedback. Similar results were found by Mesquita et al. (2008), Carreiro da Costa et al. (1996) and Rosado et al. (2008).

The retention of feedback by the athletes in this study presented a mean of $57.02 \%$. Similar results were obtained by Rosado et al. (2008), where mean retention equaled $61.9 \%$. Mesquita et al. (2008) and Januário et al. (2006) found a higher mean value of retention, $68.7 \%$ and $68.4 \%$, respectively.

Considering the effects of the athletes' characteristics on the retention process, divergent results have been found. In the present study, we found significant differences between levels of retention according to athletes' gender (the male group presented higher values of feedback retention), but this was not the case in the
Mesquita et al.'s (2008) study which revealed better retention by female participants.

In the present study, the athletes' sports level significantly influenced retention of information reproduced by athletes. Consequently, athletes with a higher sports level displayed higher values of retention. Similar results were presented by Rosado et al. (2008), where the authors reported that athletes with a higher sports level retained more ideas.

Globally, in this study, the variables related to the athletes' perception, namely acceptance of the information and the task motivation level, positively influenced retention of information.

The athletes' motivation level was associated with feedback retention as athletes with a higher motivation level displayed a lower level of FB retention. This evidence should be subject to further research, but part of the explanation may lie in the relationship between a higher task motivation level and mental effort or concentration (Nideffer, 1995). A high level of task 
motivation, sometimes enthusiastic, means a high involvement in the motor activity itself and a particular focus on the stimuli that come from the activity. This strong engagement with the task may impair the retention of FB information. The fact that athletes are overloaded by internal or external stimuli associated with soccer practice (Nideffer, 1995) might actually reduce their retention of external stimuli that come from external sources such as coaches' feedback and may affect retention of those external stimuli, namely retention of FB. The relationships between the level of motivation (task and ego motivation) and cognitive effort involved (such as the type of attention and concentration during instruction and practice) should be examined in future studies. In conclusion, the effectiveness of FB is a result of a transactional process between the coaches' instructions and athletes active processing of information. The athletes' perception and evaluation should be considered as a vital means of better coaching. More specifically, both instructional and motivational tasks should be considered to enhance skill acquisition. Contextual variables such as the type of motor tasks should also be considered as a key element.

It is suggested that future research should continue to examine other factors that may affect retention of instructions, namely contextual, socio-cognitive and emotional determinants. Thus, qualitative studies should be conducted in addition to quantitative ones to ensure a thorough understanding of athletes' information processing of feedback and other instructional information.

\section{Acknowledgments}

Thanks to Portuguese Government for their helping and supporting in this research (SFRH/BD/39578/2007). Also the collaboration from Almería University, Spain, has been very important and useful.

\section{References}

Anderson R. Control of student mediating processes during verbal learning and instruction. Review of Educational Research, 1970; 40(3): 349-369

Badzinski DM, Gill MM. Discourse features and message comprehension. In. S. Deetz (Ed.) Communication Yearbook 17. Newbury Park, CA: Sage, 301-332; 1994

Bandura A. Social foundations of thought and social action: a social cognitive theory. Englewood Cliffs, New Jersey: Prentice-Hall; 1986

Carreiro da Costa F, Marques da Costa A, Dinis J, Piéron M. An Analysis of the quality of the feedback. In C. Amade-escot, JP Barrué, JC, Bos F, Dufor M, Dugrand A. Terrise (Eds.). Recherches en EPS: Bilanet Perspectives. Editions Revue EPS, 215-223; 1998

Carreiro da Costa F, Quina J, Dinis J, Piéron M. Pedagogical Feedback: Analysis of the information referred by the student in physical education sessions. Reveu de l'Education physique, 1996; 36(2): 75-82

Clemente FM, Santos M, Lourenço FM, Dias G, Mendes R. Interpersonal Dynamics: 1v1 Sub-Phase at Sub-18 Football Players. J Hum Kinet, 2013; 36: 181-191

Cloes M, Knoden A, Piéron M. Memorization of the technical information retained during the sportive activities in controlled situations. Proceedings of the IV Journées d'Automne de L'ACAPS. Lille: Presses Université de Lille. (In French: English abstract), 222-223; 1991

Cloes M, Moreuax A, Piéron M. Students retention of teacher's feedback in physical education sessions. [Abstract] AIESEP World Convention, Moving Towards Excellence, Loughborough-England, 1990; 40

Davis FD. Perceived Usefulness, Perceived Ease Of Use, And User Acceptance Of Information Technology. MIS Quarterly, 1989; 13(3): 319-340

Doyle W. Paradigm in research of teachers' effectiveness. In Crahay Mand Lafontaine D (Eds), L'artet la 
science de l'enseignement. Bruxelles: Labor. (In French: English abstract); 435-481; 1986

Fleiss J. Statistical methods for rates and proportion (2nd ed.). New York: John Wiley; 1981

Gagné ED. The cognitive psychology of school learning. Boston: Little, Brown; 1985

Hattie J, Timperley H. The power of Feedback. Review of Educational Research, 2007; 77(1): 81-112

Januário N, Rosado A, Mesquita I. Student's retention of information and justice perceptions according discipline control. Revista Portuguesa de Ciências do Desporto, 2006; 5(2): 294-304. (In Portuguese: English abstract)

Magill RA. Motor learning: concepts and applications (4thed.). Dubuque: WCB Brown and Benchmark; 1993

McCroskey JC, Richmond VP. Foundations of human communication: An interpersonal perspective. Prospect Heights, IL: Waveland Press; 1996

Mesquita I, Rosado A, Januário N, Barroja E. Athlete's Retention of Coach's Instruction before a Judo Competition. Journal of Sport Science and Medicine, 2008; 7(3): 402-407

Nideffer RM. Test of attentional and interpersonal style revised. New Berlin, WI: Assessment Systems International; 1995

Oleto R. Perception of quality of information. Ciência da Informação, 2006; 1(35): 57-62

Rosado A, Mesquita I, Breia I, Januário N. Athlete's Retention of Coach's Instruction on Task Presentation and Feedback. International Journal of Performance Analysis in Sport, 2008; 1(8): 19-30

Siekanska M, Blecharz J, Wojtowicz A. The Athlete's Perception of Coaches' Behavior Towards Competitors with a Different Sports Level. J Hum Kinet, 2013; 39: 231-242.doi: 10.2478/hukin-2013-0086

Swalus P, Carlier G, Renaud JP. Feedback during the learning of motor tasks and their perception by students. STAPS, 1991; 12(24): 23-35

Tabachnick BG, Fidell LS. Using multivariate statistics (2nd ed.). New York: Harper, Row; 1989

Van Der Mars H. Observer reliability: issues and procedures. In P.W. Darst, DB Zakrajsek, VH Mancini (Eds.). Analyzing physical education and sport instruction. Champaign, IL: Human Kinetics; 53-79; 1989

\section{Corresponding author:}

\section{José Gallego}

Facultad de Educación,

Universidad de Almería. Ctra. De Sacramento, s/n 04120. La Cañada de San Urbano, Almería, España

Phone: 34-950 015227

Fax: 34-950 015750

E-mail: jgallego@ual.es 